\title{
Wallenberg Syndrome Caused by Radiation-Induced Brain Injury: Case Report
}

\begin{abstract}
Huan L, Yi L and Ying P*
Department of Neurology, Sun Yat-Sen University, China

*Corresponding author: Ying P, Department of

Neurology, Sun Yat-sen Memorial Hospital, Sun Yat-Sen University, Number 33, Yingfeng Road, Haizhu District,

Guangzhou City 510288, Guangdong Province, China
\end{abstract}

Received: October 28, 2016; Accepted: December 28, 2016; Published: J anuary 02, 2017

\begin{abstract}
Radiation-induced brain injury, being a serious complication after radiotherapy, is a disease of nervous system damage caused by radiation therapy for patients with head and neck cancer. Since radiation therapy can cause large blood vessel stenosis or blockage, when it damages the brain stem, Wallenberg syndrome may appear.

We report a case of Wallenberg syndrome induced by radiation therapy.

Wallenberg syndrome caused by radiation-induced brain injury is easy for clinicians to miss diagnosis or misdiagnose. Early head MR examination plays a pivotal role in reduce its occurrence rate. Clinicians should be vigilant of the blocked vascular caused by radiation therapy and initiate early thrombolysis, vascular angioplasty or stenting to make the patients get better prognosis.
\end{abstract}

Keywords: Wallenberg syndrome; Radiation-induced brain injury; Miss diagnose; Misdiagnose

\section{Abbreviations}

NPC: Nasopharyngeal Carcinoma; CT: Computed Tomography; CTA: Computed Tomography Angiography; MRI: Magnetic Resonance Imaging; FLAIR: Fluid-Attenuated Inversion Recovery

\section{Case Presentation}

Radiation-induced brain injury, being a serious complication after radiotherapy, is a disease of nervous system damage caused by radiation therapy for patients with head and neck cancer [1]. Since radiation therapy can cause large blood vessel stenosis or blockage [2], when it damages the brain stem, Wallenberg syndrome may appear. As we know, there is few report about the ischemic stroke caused by radiation-induced brain injury, and here we report a case of Wallenberg syndrome induced by radiation therapy.

A 65-year-old man presented with a 9-day history of dizziness, numbness of the left limb, vomit and walking to the right of dumping, and 1-day history of dysphagia. Seven years earlier, he had been diagnosed as stage T3N1M0 undifferentiated type of nasopharyngeal carcinoma (NPC). He had received chemotherapy with Cisplatin for 6 times over 6 weeks, and external radiation therapy to the nasopharynx with a total dose of $70 \mathrm{~Gy}$ in 35 fractions over 53 days and a supplemental dose of 60 Gy to the neck in 30 additional fractions over 46 days. There was no recurrence of NPC, but his hearing gradually dropped and muscles in the neck became stiff after radiation therapy. There was no history of particular diseases which might cause stroke before. He was diagnosed as radiationinduced temporal lobe necrosis with the result of a brain computed tomography (CT) scan during his first visit to the hospital, receiving some symptomatic treatment which made dizziness ease but left limb numbness symptoms still remain. In the ninth day, he got a severe dizziness with a new symptom of dysphagia.

The physical and neurologic examinations showed that his blood pressure was $174 / 97 \mathrm{mmHg}$, the right pupil measured $2.5 \mathrm{~mm}$, the left pupil measured $3.0 \mathrm{~mm}$, both pupils were sensitive to light, the right side of the nose was slightly shallow, the tongue extended to the right and pharyngeal reflex disappeared. CT scan of the brain (Figure 1A) showed radiation-induced bilateral temporal lobe lesions with no abnormalities in the brain stem. Cervical computed tomography angiography (CTA) (Figure 1B) was performed after neck vascular ultrasound indicating that the right vertebral artery may be embolized, and the right vertebral artery was proved to be occluded by the CTA. The contrast-enhanced magnetic resonance imaging (MRI) and fluidattenuated inversion recovery (FLAIR) (Figure 1C-1I) demonstrated an acute infarction in the right medulla and radiation-induced brain injury in bilateral temporal lobe.

After his admission to the hospital, he got the treatment of antiplatelet agents, neuroprotective drugs and other symptomatic support treatment. Fifty days later, the patient was out of the hospital with a nasogastric tube, and neurologic examination showed that pharyngeal reflex disappeared.

Informed consent was obtained from the patient and this case study was approved by the hospital ethical committee.

\section{Discussion}

To our knowledge, this is the first case report of Wallenberg syndrome caused by radiation-induced brain injury.

The patient's brain CT scan did not show the brain stem lesions in his first visit to a hospital. With the progress of the disease, the medullary infarction was finally demonstrated by the contrastenhanced MRI, which still had not been observed by the second CT scan of the brain. In fact, the patient was likely to have been at the early stage of cerebral infarction at his first visit to the hospital according to his symptoms. However, due to the limitations of brain CT in the diagnosis of brain stem lesions and the diversity of the symptoms of 


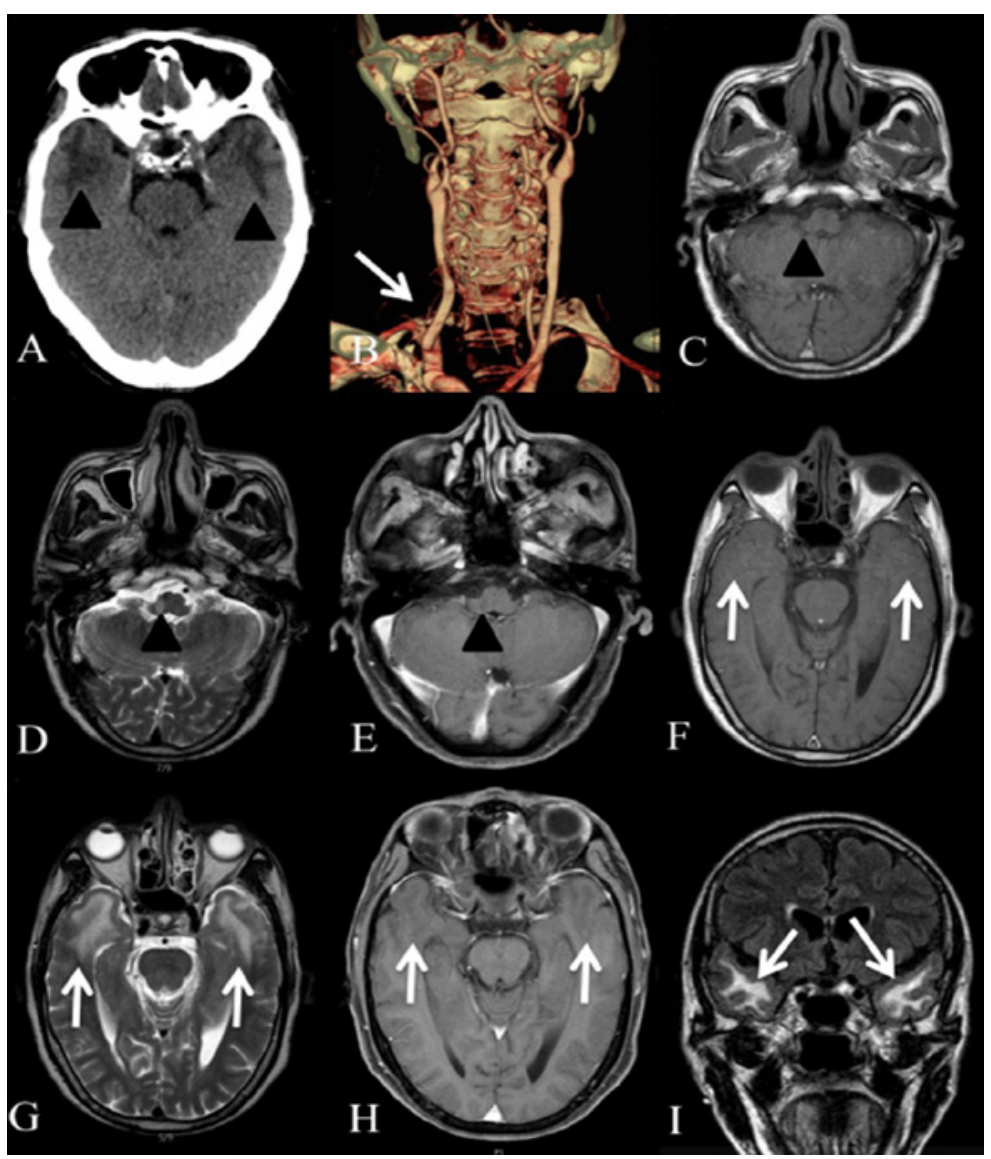

Figure 1: CT, CTA, MRI of Wallenberg syndrome caused by radiation-induced brain injury. Brain CT (A) shows radiation-induced bilateral temporal lobe lesions (arrowheads) with no abnormalities in the brain stem. Cervical CTA (B) suggests that the right vertebral artery is occluded (arrow). The contrast-enhanced MRI and FLAIR (C-I) demonstrates an acute infarction in the right medulla (arrowheads) and radiation-induced brain injury in bilateral temporal lobe (arrows) (C and F are non-contrast T1 weighted images, D and G T2 weighted images, E and H contrast-enhanced T1 weighted images and I FLAIR).

CT: Computed Tomography; CTA: Computed Tomography Angiography; MRI: Magnetic Resonance Imaging; FLAIR: Fluid-Attenuated Inversion Recovery.

radiation-induced brain injury, clinicians might miss diagnosis or misdiagnose. This case report reminds us that if brain stem lesions are suspected, the head MRI examination should be done as early as possible to get the best treatment period for patients.

In addition, the patient has the right vertebral artery occlusion. Various radiation damage to the cervicocerebral arteries after head and neck irradiation have already been documented, presenting the most common increased intima media thickness [3,4]. Moreover, a study confirmed that the cause of the posterior cerebral artery ischemia is the occlusion of the vertebral artery or the beginning of the vertebral artery recently. As the medullary branches of the posterior inferior cerebellar artery are end branches, ischemic changes in the dorsal lateral of the medulla are caused by vertebral artery occlusion [5]. Since the patient had no history which might induce stroke, we strongly speculate the symptom rather rendered by radiation therapy than other causes. Although some treatment of ischemic stroke and radiation-induced brain injury is similar such as rehabilitation training [6], thrombolysis and interventional therapy are not routine treatment for radiation-induced brain injury. Given early diagnosis, clinicians might use thrombolysis, vascular angioplasty or stenting to make the patient get better prognosis before the vascular completely blocked.
In conclusion, Wallenberg syndrome caused by radiation-induced brain injury is easy for clinicians to miss diagnosis or misdiagnose. Early head MR examination plays a pivotal role in reduce its occurrence rate. Clinicians should be vigilant of the blocked vascular caused by radiation therapy and initiate early thrombolysis, vascular angioplasty or stenting to make the patients get better prognosis.

\section{Acknowledgment}

This study is supported by Project of Product, Study and Research of Guangzhou city (No. 201508020058 to Y.P).

\section{References}

1. Li $Y$, Shi $X$, Rong $X$, Peng $Y$, Tang $Y$. Neurosurgery and prognosis in patients with radiation-induced brain injury after nasopharyngeal carcinoma radiotherapy: a follow-up study. Radiat Oncol. 2013; 8: 88.

2. Favre JP, Nourissat A, Duprey A, Nourissat G, Albertini JN, Becquemin JP. Association Universitaire de Recherche en Chirurgie Vasculaire. Endovascular treatment for carotid artery stenosis after neck irradiation. J Vasc Surg. 2008; 48: 852-858.

3. So NM, Lam WW, Chook P, Woo KS, Liu KH, Leung SF, et al. Carotid intimamedia thickness in patients with head and neck irradiation for the treatment of nasopharyngeal carcinoma. Clin Radiol. 2002; 57: 600-603.

4. Dorresteijn LD, Kappelle AC, Scholz NM, Munneke M, Scholma JT, Balm AJ, et al. Increased carotid wall thickening after radiotherapy on the neck. Eur J Cancer. 2005; 41: 1026-1030. 
5. Kim JS. Pure lateral medullary infarction: clinical-radiological correlation of 130 acute, consecutive patients. Brain. 2003; 126: 1864-1872.

6. Tang Y, Shen Q, Wang Y, Lu K, Wang Y, Peng Y. A randomized prospective study of rehabilitation therapy in the treatment of radiation-induced dysphagia and trismus. Strahlenther Onkol. 2011; 187: 39-44.
Austin J Cerebrovasc Dis \& Stroke - Volume 4 Issue 1 - 2017

ISSN : 2381-9103 | www.austinpublishinggroup.com

Ying et al. @ All rights are reserved
Citation: Huan L, Yi L and Ying P. Wallenberg Syndrome Caused by Radiation-Induced Brain Injury: Case Report. Austin J Cerebrovasc Dis \& Stroke. 2017; 4(1): 1050. 